\title{
CAMARÕES DE ÁGUA DOCE DO BRASIL: DISTRIBUIÇÃO GEOGRÁFICA
}

PETRONIO ALVES COELHO 1 MARILENA RAMOS-PORTO 2

\begin{abstract}
The authors have studied the geographical distribution of 33 freshwater prawn species found in Brazil and surrounding areas, as part of a research project which views the development of a national technology for the freshwater prawn culture. The fauna comprises litoral species found in lowlands along the coast, in areas limited at the south by the $15^{\circ} \mathrm{C}$ isotherm for the average air temperature of the coldest month, and continental species, which can be separated in tropical, subtropical and temperature ones, whose occurrence areas were delimited by the $20^{\circ} \mathrm{C}$ and $15^{\circ} \mathrm{C}$ average air temperature of the coldest month.
\end{abstract}

\section{INTRODUÇÃO}

Há vários anos foi iniciado, no Departamento de Oceanografia da UFPE, um estudo sobre os camarões de água doce, com o objetivo de desenvolver uma tecnologia adequada para o cultivo das espécies nativas. Como parte desta pesquisa foram estudadas as áreas de ocorrência das espécies, e as condições ambientais características do habitat destes animais. Este conhecimento é de importância, permitindo a previsão do êxito do cultivo de espécies nativas nas diversas partes do país.

Os autores agradecem a colaboração das numerosas pessoas e entidades que contribuíram para a realização da pesquisa em suas diversas fases. $O$ CNPq apoiou financeiramente a realização de 3 expedições às águas interiores, e a FINEP também deu seu apoio à pesquisa, financiando 2 expedições às águas interiores, assim como visitas a instituições de pesquisas de outros Estados, tornando possível a obtenção de dados existentes em suas coleções. Colegas de várias instituições permitiram o estudo de material sob sua custódia, principalmente os professores Gustavo A. S. Melo (Museu de Zoologia da USP) e Alceu Lemos de Castro (Museu Nacional, UFRJ). Os autores agradecem em particular aos Departamentos de Oceanografia da UFPE e de Pesca da UFRPE, que possibilitaram a realização destas pesquisas com o apoio necessário.

\section{MATERIAL E METODOS}

O estudo da distribuição geográfica das espécies está baseado na análise de dados provenientes de várias fontes: informações contidas na literatura (Anexo I), dados de coleta do material depositado nas coleções dos Departamentos de Pesca da UFRPE e Oceanografia da UFPE, do Museu Nacional (Rio de Janeiro) e Museu de Zoologia (São Paulo).

As espécies foram classificadas como "litorais" e "continentais", de acordo com os critérios estabelecidos por Rodríguez (1981). As bacias hidrográficas foram divididas em costeiras e interiores. As costeiras foram agrupadas em: I) Bacias do Norte (Amapá, Pará e Maranhão, exceto o Rio Parnaíba); II) Bacias do Nordeste (Piauí, Ceará, Rio Grande do Norte, Paraíba, Pernambuco, Alagoas, Sergipe e Bahia); III) Bacias do Sudeste (Espírito Santo, Minas Gerais, Rio de Janeiro, São Paulo, Paraná e Santa Catarina); IV) Bacias do Sul (Rio

1. Departamento de Oceanograria, Universidade Federal de Pernambuco. Pesquisador do CNPq. CNPq.

. Departaments de Pesca, Universidade Federal Rural de Pernambuco. Pesquisadora do 
Grande do Sul). As bacias interiores foram subdivididas da seguinte forma: A) Bacia do Amazonas (excluindo o Baixo Amazonas); B) Bacia do São Francisco (à montante da Cachoeira de Paulo Afonso) C) Bacia do Paraguai; D) Bacia do Paraná; E) Bacia do Uruguai (Fig. 1).

Os dados existentes sobre altitude e clima dos locais de coleta foram analisados, permitindo a compreensão de sua influência sobre a distribuição das espécies.

\section{RESULTADOS}

Distribuição das espécies litorais:

Atya scabra, Potimirim potimirim, P. glabra, Palaemon pandaliformis, Macrobrachium heterochirus, $M$. olfersii, $M$. carcinus e $M$. acanthurus são encontrados apenas nas bacias costeiras e no curso inferior dos grandes rios, geralmente ao norte de $28^{\circ} \mathrm{S}$. Seu limite sul está localizado nas proximidades da isoterma de $15^{\circ} \mathrm{C}$ para a temperatura média do ar no mês mais frio. No entanto, Buckup \& Bond (1982) registraram a ocorrência de $M$. olfersii e $P$. pandaliformis no Rio Grande do Sul. A altitude dos locais de coleta normalmente não ultrapassou a isoípsa de 100 metros.

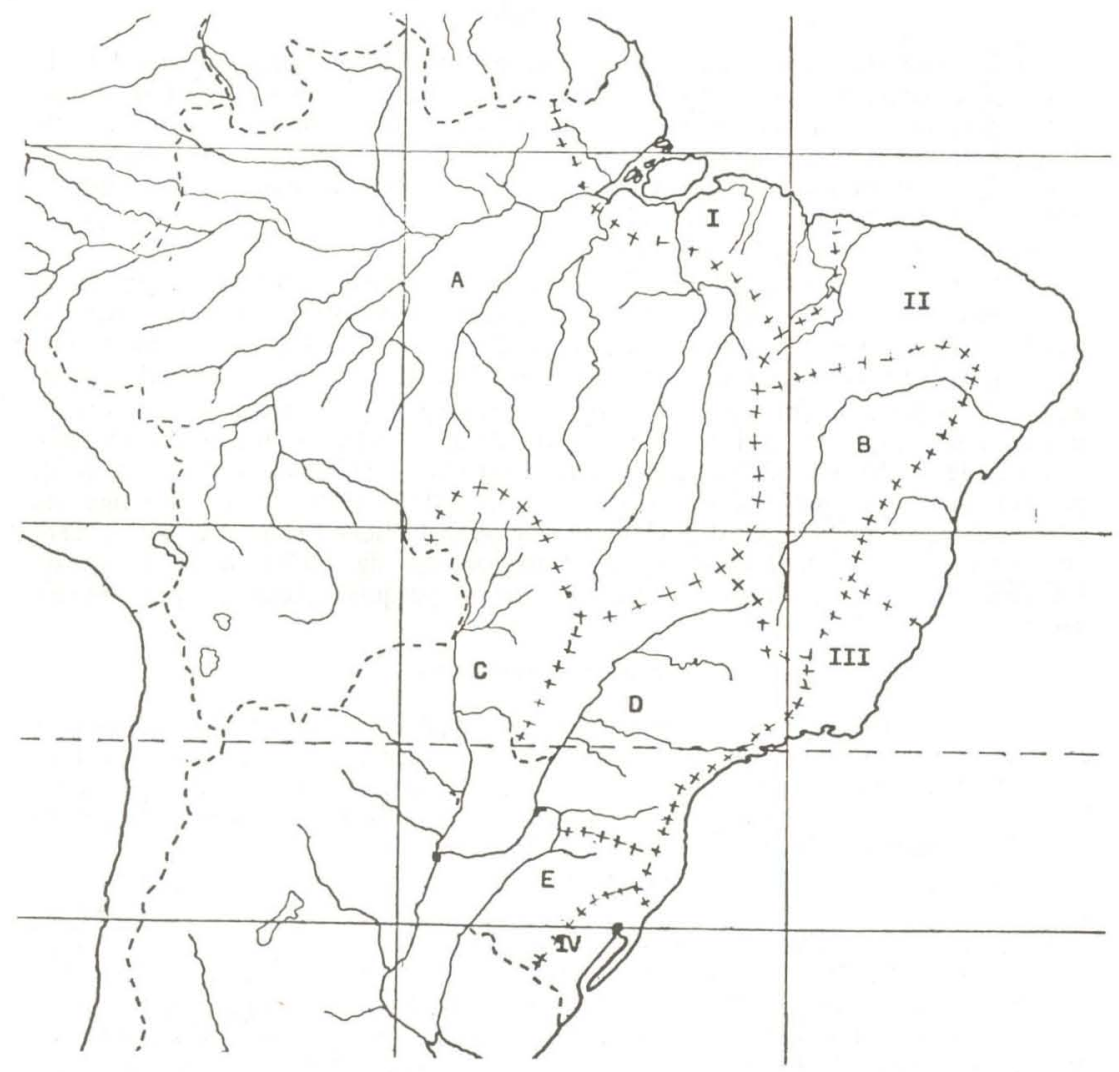

Fig. 1 - Bacias hidrográficas do Brasil. Bacias costeiras: I. Norte; II. Nordeste; III. Sudeste; IV. Sul. Bacias interiores: A. Amazonas; B. São Francisco; C. Paraguai; D. Paraná; E. Uruguai. 
Existem apenas uns poucos dados sobre a temperatura da água no momento da coleta. $P$. potimirim e $M$. acanthurus foram encontrados em água com temperatura superior a $30^{\circ} \mathrm{C}$ no momento da coleta, e $M$. acanthurus e $P$. pandaliformis foram recolhidos em água com temperatura entre 18 e $21^{\circ} \mathrm{C}$; estes valores possivelmente estão próximos dos limites extremos de temperatura da água onde vivem estes camarõ்es.

\section{Distribuição das espécies continentais}

A distribuição das espécies continentais é mostrada nas tabelas I e II. Macrobrachium amazonicum, $M$. nattereri, $M$. jelskii e $M$. brasiliense possuem áreas de ocorrência muitos vastas, equivalendo a grande parte do território estudado, enquanto as demais espécies ocupam parcelas menores no interior deste vasto território. De um modo geral, as áreas de ocorrência estão contidas dentro dos limites da isoterma de $20^{\circ} \mathrm{C}$ para a temperatura média do ar do mês mais frio; fazem exceção $M$. nattereri, $E$. burchelli e $A$. paraguayensis que, em alguns pontos, ultrapassam este limite. Todas as espécies que ocorrem junto com $M$. amazonicum possuem, além disto, área de ocorrência totalmente incluída na região limitada pela isoterma de $25^{\circ} \mathrm{C}$ para a temperatura média do ar para o mês mais quente. Ocorrem todas em locais de baixa altitude, porém $M$. amazonicum, $M$. jelski e talvez alguma outra espécie ultrapassam o limite de 400 metros de altitude. A área de ocorrência destas espécies corresponde à porção de clima tropical compreendendo, no Brasil, as bacias do Amazonas, São Francisco, costeiras do Norte e Nordeste, assim como do Paraná (das vizinhanças de Urubupungá para montante) e do Paraguai.

Macrobrachium potiuna e $M$. iheringi, de uma parte, e $M$. borellii, Pseudopalaemon bouvieri e Palaemonetes argentinkis, de outra parte, constituem dois grupos de espécies com áreas de ocorrência distintas, que entram em contacto entre si, ou com as espécies mencionadas no parágrafo anterior apenas em determinadas localidades. Os territórios ocupados por M. potiuna e $M$. iheringi estão contidos ao norte pela isoterma de $20^{\circ} \mathrm{C}$ em julho e ao sul pela isoterma de $10^{\circ} \mathrm{C}$ no mesmo mês; estas espécies têm sido coletadas em localidades de altitude variando entre poucos metros até as proximidades de 1.200 metros. As espécies restantes, isto é, $M$. borellii, $P$. bouvieri e $P$. argentinus habitam localidades com a temperatura média do ar do mês mais frio não ultrapassando $15^{\circ} \mathrm{C}$ (em algumas localidades $M$. borellii ultrapassa este limite em direção ao norte); $M$. borellii pode ser encontrado na Argentina em localidades com altitude superior a 1.200 metros, $P$. argentinus não ultrapassa a isoípsa de 400 metros e $P$. bouvieri está restrito a terras baixas, de altitude inferior a 100 metros.

Restam ainda duas espécies conhecidas apenas de territórios muito restritos: Cryphiops brasiliensis (Distrito Federal) e Macrobrachium holthuisi (São Paulo); a primeira é encontrada em altitudes bastante elevadas e a segunda em terras baixas, porém com a temperatura média no mês de julho entre 15 e $20^{\circ} \mathrm{C}$.

As áreas de ocorrência de $M$. potiuna, $M$. iheringi, $M$. holthuisi e $C$. brasiliensis correspondem a um setor subtropical que abrange as bacias costeiras do Sudeste e parte da Bacia do Paraná, se estendendo para o norte até o Distrito Federal e regiões adjacentes e para o sul até o Rio Grande do Sul.

Por outro lado, $M$. borellii, $P$. bouvieri e $P$. argentinus habitam o setor temperado, correspondendo no Brasil à Bacia do Uruguai e outros trechos do Rio Grande do Sul.

Existem apenas alguns dados sobre a temperatura da água no momento da coleta dos camarões; $M$. amazonicum e $M$. jelskii foram encontrados até em água com temperatura superior a $36^{\circ} \mathrm{C}$ no momento da coleta, e $M$. potiuna e $C$. brasiliensis foram coletados em água com temperatura entre 15 e $21^{\circ} \mathrm{C}$. 
Distribuição das espécies continentais tropicais

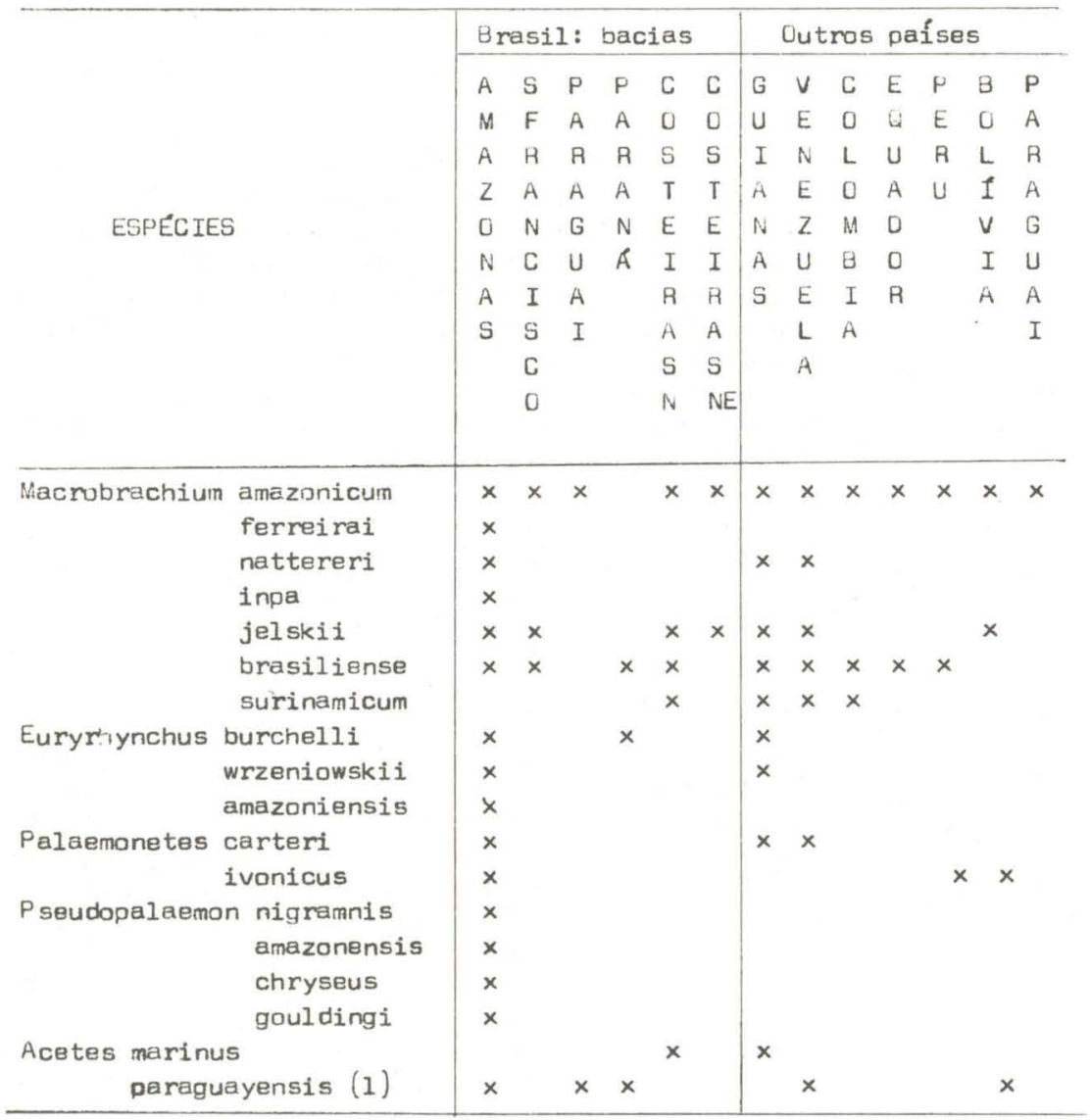

(1) Também na Argentina

\section{COMENTÁRIOS}

Tem havido certas controvérsias acerca da divisão da Região Neotrópica em subregiões e províncias, porém a maioria dos autores reconhece a existência da subregião Brasiliana, correspondente à vertente atlântica da América do Sul desde a Bacia do Madalena, na Colômbia, até a Bacia do Rio da Prata, na Argentina. Esta subregião tem sido dividida .em um número variável de províncias, não havendo unanimidade de opinião entre os autores. Zajciw (1971) é de opinião de que existem duas faunas distintas na subregião Brasiliana: uma meridional e outra setentrional. Este esquema pode ser adotado 
Distribuiçao das espécies Gontinentais não tropicais

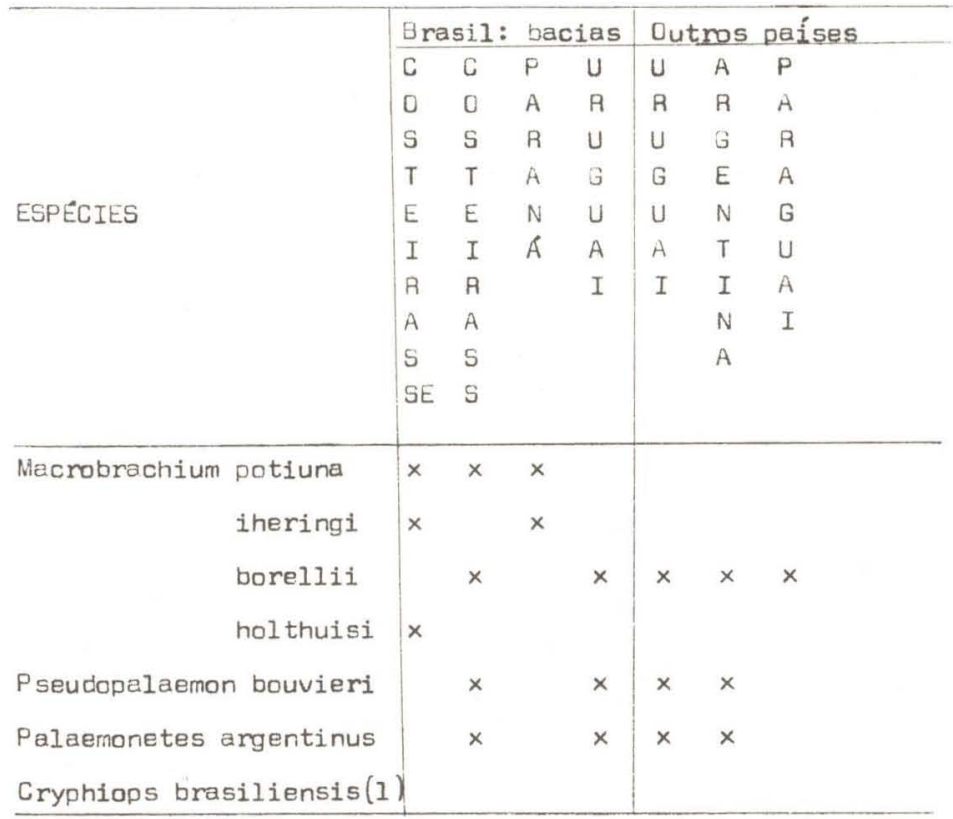

(1) Distrito Federal

para os camarões de água doce, porém os dados expostos sugerem que a temperatura tenha influência na distribuição das espécies. Neste sentido, as áreas das espécies tropicais, subtropicais e temperadas estariam delimitadas, em grande parte, pelas temperaturas médias do ar do mês mais frio.

\section{REFERENCIAS}

Buckup, L. \& Bond, G., 1982. Palaemonidae de águas continentais do Rio Grande do Sul e Santa Catarina, Brasil (Crustacea, Decapoda, Palaemonidae). Res. IX Congr. bras. Zool., Porto Alegre: 193.

Rodríguez, G., 1981. Decapoda, In: Hurlbert, S. H., Rodríguez, G. \& Santos, N. D., eds., Aquatic Biota of Tropical South America. Part 1: Arthropoda. San Diego State University, San Diego, California, p. 41-51.

Zajciw, D., 1971. Algumas considerações a respeito dos assuntos zoogeográficos no Brasil. Arq. Mus. nac., Rio de J. 54: 243-247. 


\section{ANEXO I}

Bibliografia consultada sobre a ocorrência de camarões na área estudada.

Coelho, P. A., Koening, M. L. \& Ramos, M. A., 1979. A macrofauna bêntica dos estuários de Pernambuco e da Paraíba. Actas IV Congr. latinoam. Zool., Caracas 2: 497-528.

Coelho, P. A. \& Ramos, M. A., 1973. Contribuição ao conhecimento dos camarões comerciais do Norte e Nordeste do Brasil. XXXVIII Comissâo oceanogr. N. Oc. Almirante Saldanha (8/11 a 20/12/1968), p. 123-131.

Genofre, G. C. \& Lobão, V. L., 1978. Macrobrachium holthuisi sp. n., a new species of shrimp (Decapoda, Macrura). Crustaceana, Leiden 35: 273-276.

Gomes-Correa, M. M., 1973. Descrição de uma espécie nova do gênero Cryphiops (Decapoda: Natantia: Palaemonidae). Revta bras. Biol., Rio de J 33: 169-173.

Gomes-Correa, M. M., 1977. Palaemonídeos do Brasil (Crustacea, Decapoda, Natantia. Dissertação de Mestrado, Universidade Federal do Rio de Janeiro, $191 \mathrm{pp}$.

Holthuis, L. B., 1959. The Crustacea Decapoda of Suriname (Dutch Guiana). Zool. Verhandel., Leiden 44: 1-296.

Holthuis, L. B., 1961. A general revision of the Palaemonidae (Crustacea, Decapoda, Natantia) of the Americas. I. The subfamilies Euryrhynchinae and Pontoniinae. Occas. Papers Allan Hancocy Found. 11: 1-332.

Holthuis, L. B., 1962. Idem. II. The subfamily Palaemoninae. Ibidem 12: 1-396.

Holthuis, L. B., 1966. A collection of freshwater prawns (Crustacea, Decapoda, Palaemonidae) from Amazonia, Brazil, collected by Dr. G. Marlier. Bull. Inst. r. Sci. nat. Belg. 42: 1-11.

Kensley, B. Z. Walekr, I., Palaemonid shrimps from the Amazon Basin, Brasil (Crustacea: Decapoda: Natantia). Smithson. Contr. Zool. 362: 1-28.

Omori, M., 1975. The systematics, biogeography and fishery of epipelagic shrimps of the genus Acetes (Crustacea, Decapoda, Sergetidae). Bull. Ocean Res. Inst. Univ. Tokyo 7: 1-91.

Ramos-Porto, M., 1980. Estudo ecológico da região de Itamaracá, Pernambuco, Brasil. VIII. Crustáceos decápodos natantes. Trabalhos oceanogr. Univ. Fed. Pernambuco, Recife 15: 277-310.

Ramos-Porto, M. \& Palacios, J. A. P., 1983. Estudo ecológico do Rio Capibaribe-Mirim, Pernambuco, Brasil. IV. Crustáceos decápodos natantes. Ibidem 16: 265-296.

Ringuelet, R., 1949. Camarones y cangrejos de la zona de Goya (Sergestidae, Palaemonidae y Trichodactylidae). Notas Mus. La Plata 14(119): 79-109.

Rodríguez, G., 1982. Fresh-water shrimps (Crustacea, Decapoda, Natantia) of the Orinoco Basin and the Venezuelan Guayana. J. Crust. Biol 2(3): 378-391.

Tiefenbacher, L., 1978. Zur Systematik and Verbreitung der Euryrhynchinae (Decapoda, Natantia, Palaemonidae). Crustaceana, Leiden 35: 177-189.

Villalobos F., A., 1969. Contribución al conocimiento de los Atyidae de Mexico. II. (Crustacea, Decapoda). Estudio de algunas especies del género Potimirim (= Ortmannia), con descripción de una especie nueva en Brasil. Anal. Inst. Biol. México 30: 269-330. 